\title{
Clinical use of photobiomodulation as a supportive care during radiation therapy
}

\author{
Guillaume Klausner $^{1} \cdot$ Idriss Troussier $^{2,3} \cdot$ Charles-Henry Canova $^{2} \cdot$ René-Jean Bensadoun $^{2}$
}

๑) Springer-Verlag GmbH Germany, part of Springer Nature 2021

Keywords Photobiomodulation $\cdot$ Low-level light therapy $\cdot$ Radiation therapy $\cdot$ Neoplasms $\cdot$ Stomatitis $\cdot$ Radiation dermatitis $\cdot$ Lymphedema

\section{Introduction}

Photobiomodulation (PBM), formerly called "low-level laser therapy (LLLT)," is a non-invasive therapy used, among other indications, as a preventive or curative treatment in the management of several radiotherapy side effects. The common definition of PBM is "a mechanism by which nonionizing optical radiation in the visible or near-infrared spectral range is absorbed by endogenous chromophores to trigger photophysical and photochemical events without causing thermal damage, resulting in physiological changes and therapeutic benefits" [1]. The wavelengths used in PBM range from red light (600-700 nm (nm)) to infrared $(700-100 \mathrm{~nm})$ and the therapeutic dose is the energy density measured in joules $\left(\mathrm{J} / \mathrm{cm}^{2}\right)$ [2]. Historically, PBM was developed by NASA using single low-intensity laser beams to accelerate the regeneration of astronauts' muscle cells [3]. Since the first findings in the 1960s, lasers have been the most used and studied device as PBM modality. The advantages of laser beams are that they are highly concentrated, collimated, monochromatic electromagnetic wave beams and have a limited frequency range, long coherence length with the possibility of high-power densities [4]. Recent years have seen the development of devices using non-coherent light sources, such as light-emitting diodes (LEDs) or broadband

René-Jean Bensadoun

renejean.bensadoun@che-nice.com

1 Oncology - Radiotherapy, Pierre and Marie Curie University, Paris Sorbonne University, 91-105 Boulevard de l'Hôpital, 75013 Paris, France

2 Oncology - Radiotherapy, Centre de Haute Énergie (CHE), 10 Boulevard Pasteur, 06000 Nice, France

3 Radio-Oncology, University Hospitals of Geneva (HUG), Rue Gabrielle-Perret-Gentil 4, 1205 Geneva, Switzerland lamps. LED machines have become increasingly important in the scientific literature and are now common in clinical practice. The advantages of LEDs are mobility of the device, ability to treat a large area at once, safety, and being more economical with a lower cost per $\mathrm{mW}$ than lasers [4].

The biological rationale of PBM is the transfer of incident photon energy to an intracellular target which then affects cellular metabolism. The main target of PBM is the mitochondria. Absorption of red to infrared light waves by the mitochondria stimulates its role in the respiratory cycle chain of electron transport. This results in direct activation of cytochrome $\mathrm{C}$, a direct increase in ATP and nitric oxide (NO) production, and expression and transcription of genes involved in cell replication signaling pathways [5]. NO is a vasodilator that increases the perfusion of tissues with oxygenated blood, but also of lymphatic vessels that become dilated and less porous. In addition, PBM induces increased production of pro-collagen and growth factors, including vascular endothelial growth factors and fibroblast growth factors [6].

Recently, the indications and modalities of PBM in the management of cancer treatment-induced toxicities were the subject of international recommendations at the 12th World Association of Photobiomodulation Therapy (WALT) Conference in 2018 [2, 7, 8]. Our objective is to describe and discuss the current and potential indications in clinical practice of PBM as a supportive care for patients treated by radiotherapy or chemoradiotherapy, based on our clinical experience and available guidelines and published literature. 


\section{Acute oral mucositis}

Oral mucositis is an extremely common side effect. The cause of oral mucositis may be multifactorial, involving chemotherapy, radiotherapy, molecular targeted therapy, or even immunotherapy. In addition to its functional impact and obvious impairment of quality of life, mucositis is a risk factor for bacteremia and sepsis or chronic mucositis [9]. Oral mucositis can also lead to a break or a dose reduction of radiotherapy or chemotherapy, and therefore loss of chance in overall survival for the patient.

The Multinational Association of Supportive Care in Cancer and International Society for Oral Oncology (MASCC/ ISOO) Mucositis Study Group identified high-level evidence studies that supported the clinical use of PBM to prevent or treat radiation-induced acute oral mucositis [10-17]. Of the eight published placebo-controlled randomized trials, only one was negative on its primary endpoint, while the other seven were positive in favor of PBM in the management of radiation-induced acute mucositis. Consistent findings across these randomized trials were a decrease in the incidence of grade $\geq 3$ acute mucositis, a decrease in pain intensity and opioid consumption, and an improvement in quality of life. Some publications also suggested a reduction in the intensity and duration of acute mucositis, a reduction in parenteral nutrition dependence, and a functional improvement, notably in salivary flow. From a methodological point of view, these eight trials were of heterogeneous quality. Indeed, the primary endpoint was not always clear or was not specified, the time at which the outcomes were assessed was not always specified or the measurements were repeated without adequate statistical methodology. Finally, the statistical tests were often multiple leading to inflation of the $\alpha$-risk and thus limiting the power of the conclusions. In contrast, validated and consensus-based scales and scores were systematically used to assess the grade of mucositis, pain intensity, or quality of life. More recently, a systematic review of the literature followed by a meta-analysis including six of these eight trials suggested that PBM may reduce the risk of severe oral mucositis during radiotherapy by $64 \%$ ( $\mathrm{HR}=0.36,95 \% \mathrm{CI}[0.29-0.44])$ and is also associated with favorable cost-effectiveness [18]. Another systematic review of the literature, which included ten placebo-controlled studies, found that nine of the ten studies favored the clinical use of PBM during radiotherapy to improve pain control and that five out of seven studies that compared analgesic drug requirements supported PBM to reduce opioid consumption [19].

To summarize, the MASCC/ISSO endorses the clinical use of PBM for the prevention of radiation-induced acute mucositis [20, 21]. The main limit is the wide variety of PBM protocols available. As such, it is recommended that the specific physical parameters of a published protocol be carefully followed to ensure optimal results (including the dose to the treated tissue over the entire area of interest). In other words, if the physical parameters of one protocol are combined with those of another, then the efficacy of the PBM will be random.

\section{Epithelitis}

Epithelitis or acute radiation dermatitis is the most common radiotherapy-related side effect. Prevention and management of acute radiodermatitis recommended by MASCC consist of daily hygiene measures and the application of topical steroids [22]. Several prospective controlled trials assessed the efficacy of PBM to reduce rates of severe epithelitis during adjuvant radiotherapy in breast cancer. The first double-blind placebo-controlled trial randomized 33 patients treated with adjuvant radiotherapy for breast cancer to either LED PBM (590 nm, $0.15 \mathrm{~J} / \mathrm{cm}^{2}, 100$ pulse, $250 \mathrm{~ms} /$ pulse, $2 \mathrm{~cm}$ from the breast skin) or a sham laser immediately before and after each radiotherapy session. The trial was negative, and no statistical difference was found between the groups [23]. More recently, the DERMIS pilot study prospectively compared two successive groups of patients treated with adjuvant radiotherapy for breast cancer. The first group received twice weekly laser PBM (808-905 nm, $0.168 \mathrm{~W} / \mathrm{cm}^{2}, 4 \mathrm{~J} /$ $\mathrm{cm}^{2}$, spot area $19.635 \mathrm{~cm}^{2}, 5 \mathrm{~cm}$ from the breast skin). The second group had routine supportive care without real or sham PBM. At the end of radiotherapy, the rate of grade $\geq 2$ epithelitis was significantly decreased in the PBM group suggesting efficacy of PBM in this setting [24]. Following this publication, the randomized phase III TRANSDERMIS trial included 120 patients treated for a breast cancer to confirm efficacy of PBM following the same protocol with a more robust methodology. At the end of radiotherapy, the rate of epithelitis grade $\geq 2$ as well as quality of life scores was significantly improved in the PBM group. The TRANSDERMIS trial was positive and thus confirmed PBM as an effective supportive care to prevent acute radiodermatitis in the setting of adjuvant breast cancer radiotherapy [25].

Regarding head and neck cancer, the prospective randomized DERMISHEAD trial evaluated efficacy of PBM to prevent acute epithelitis in this indication. The 46 patients included were randomized to either laser PBM $(808-905 \mathrm{~nm}$, $1.1-25 \mathrm{~W}, 4 \mathrm{~J} / \mathrm{cm}^{2}$, spot area $3.14 \mathrm{~cm}^{2}, 300-600 \mathrm{~s}, 2 \mathrm{~cm}$ from the skin) or a sham laser from day one of radiotherapy, twice a week. The use of PBM significantly reduced the rate of grade $\geq 2$ epithelitis at the end of radiotherapy. Therefore, the DERMISHEAD trial supported the implementation of PBM in routine practice to prevent acute epithelitis for head and neck cancer [26]. 
In summary, the available data tend to show that PBM reduces the rate and severity of acute epithelitis in patients treated with radiotherapy [27]. Further randomized clinical trials are awaited to define the best PBM protocols that can be applied in clinical routine.

\section{Acute dysphagia}

Regarding head and neck cancer, dysphagia may be present at diagnosis in two-third of patients and may persist in the mid- and long terms. In the literature, dysphagia commonly caused or promoted serious complications such as inhalation pneumonitis in up to $20 \%$ of patients [28]. In addition to direct tumoral invasion, different mechanisms are involved in dysphagia. These may coexist, and there is a close relationship between acute mucositis, xerostomia, and dysphagia.

Some prospective trials have reported efficacy of PBM to prevent dysphagia in patients treated with (chemo)radiotherapy for head and neck cancer. It should be noted that dysphagia was systematically evaluated in secondary exploratory analyses, and to date, no prospective randomized trial has evaluated the efficacy of PBM with dysphagia as the primary endpoint. A first phase III double-blind trial randomized 75 patients between treatment with laser PBM $(660 \mathrm{~nm}, 10$ $\mathrm{mW}, 2.5 \mathrm{~J} / \mathrm{cm}^{2}$, spot area $4 \mathrm{~mm}^{2}, 10 \mathrm{~s} / \mathrm{spot}$, intra-oral) or a sham laser daily before each session and throughout the duration of radiotherapy. This trial found no improvement in acute dysphagia of grade $\geq 3$ or feeding tube dependence in the PBM group [10]. A second phase III triple-blind trial randomized 221 patients between laser PBM $(632.8 \mathrm{~nm}, 24$ $\mathrm{mW}, 3.0 \mathrm{~J} / \mathrm{cm}^{2}$, spot area $1 \mathrm{~cm}^{2}, 15-20 \mathrm{~min} / \mathrm{session}$, intraoral) and a sham laser daily before each session and for the duration of radiotherapy. In contrast to the previous study, PBM decreased the incidence of acute dysphagia, total parenteral nutrition, and opioid use [11]. Finally, a third phase III double-blind trial randomized 94 patients to either laser PBM $\left(660 \mathrm{~nm}, 100 \mathrm{~mW}, 4 \mathrm{~J} / \mathrm{cm}^{2}\right.$, spot area $0.24 \mathrm{~cm}^{2}, 10 \mathrm{~s} /$ spot, intra-oral) or a sham laser daily before each session and throughout the course of radiotherapy. This trial was positive and confirmed that PBM was associated with significantly lower gastrostomy need and opioid use [15]. To confirm these preliminary results, clinical trials assessing dysphagia as a primary outcome are required.

\section{Xerostomia}

Grade $\geq 2$ xerostomia occurs in approximatively $76 \%$ of patients 3 months after radiotherapy for a head and neck cancer and in $30 \%$ of patients at 2 years in the era of intensity-modulated conformal radiation therapy [29]. Saliva is a key factor in maintaining mucosal integrity, oral wound healing, taste perception, bolus formation, swallowing, and speech [30].

Four randomized trials have evaluated efficacy of PBM in the prevention or treatment of xerostomia in patients treated with radiotherapy or chemoradiotherapy. A first trial randomized 30 patients treated with high-dose chemoradiotherapy prior to bone marrow autotransplantation to either preventive treatment with laser PBM $(632.8 \mathrm{~nm}, 60$ $\mathrm{mW}, 1.5 \mathrm{~J} / \mathrm{cm}^{2}, 10 \mathrm{~s} / \mathrm{spot}$, intra-oral, daily from d-5 to d-1 prior to radiotherapy) or conventional care. Xerostomia of any grade was statistically improved in patients treated with PBM without any significant adverse effects [31]. A second trial randomized 60 patients undergoing radiotherapy for head and neck cancer to either laser PBM $(685 \mathrm{~nm}, 35$ $\mathrm{mW}, 2 \mathrm{~J} / \mathrm{cm} 2$, spot size $0.028 \mathrm{~cm}^{2}, 25 \mathrm{~s} / \mathrm{spot}$, intra-oral) or a sham laser daily. The outcomes were statistically in favor of PBM with significantly higher salivary flow after PBM therapy [14]. Finally, very recently, a third trial randomized 21 patients undergoing radiotherapy for head and neck cancer between laser PBM with intra-oral applications $(660 \mathrm{~nm}$, $40 \mathrm{~mW}, 10 \mathrm{~J} / \mathrm{cm}^{2}$, spot area $0,028 \mathrm{~cm}^{2}, 7 \mathrm{~s} / \mathrm{spot}$, contact) and extra-oral salivary gland applications $(810 \mathrm{~nm}, 40 \mathrm{~mW}, 25 \mathrm{~J} /$ $\mathrm{cm}^{2}$, spot area $0.028 \mathrm{~cm}^{2}, 17.5 \mathrm{~s} / \mathrm{spot}$, contact) three times a week for the duration of radiotherapy or sham laser. Up to 2 months after the end of radiotherapy, no statistical differences between the two groups were observed regarding salivary flow, saliva composition, xerostomia, or quality of life [32].

In the treatment of radiation-induced xerostomia sequelae of head and neck cancer, a pilot study randomized 23 patients with severe hyposalivation to laser PBM (830 nm, $100 \mathrm{~mW}, 71 \mathrm{~J} / \mathrm{cm}^{2}$, spot area $0.028 \mathrm{~cm}^{2}, 20 \mathrm{~s} / \mathrm{spot}$ ) twice weekly for 6 weeks for a total of 12 sessions at the major salivary glands or conventional care. The trial was negative; there was no statistically significant difference between the two groups on either stimulated or unstimulated salivary flow, xerostomia, or oral health-related quality of life [33].

Recently, a systematic review of the literature of 314 articles including five controlled trials underlined the wide diversity of PBM protocols (type of laser, extra-oral or intraoral application, number of sites applied, power, time per spot, fluence) and the conflicting results published for both prevention and treatment of xerostomia induced by radiotherapy alone or in combination with chemotherapy [34]. In order to further assess the potential role of PBM in preventing radiation-induced xerostomia, a meta-analysis of summarized data from five controlled trials using PBM in this indication was conducted in 2020. The conclusion suggested an increase in unstimulated salivary flow and stimulated salivary flow after preventive treatment with $\mathrm{PBM}$, suggesting to the authors that PBM could minimize the impact of radiation-induced xerostomia without any adverse event [35]. 
In summary, the evidence-based efficacy of PBM in the prevention and management of xerostomia associated with radiotherapy is mixed and based on a low to moderate level of evidence. The general trend suggests that PBM may be of value in preventing or treating salivary gland dysfunction. Randomized placebo-controlled trials and especially precise PBM protocols are needed to ensure preliminary positive results.

\section{Lymphedema}

Lymphedema of the upper limb affects approximatively $20 \%$ of patients treated for breast cancer, resulting from alteration of lymphatic structures by surgery or radiotherapy or both [36]. The prevention of lymphedema is based on hygienic and dietary measures and the treatment on manual therapies of decongestion or lymphatic drainage [37].

A historical meta-analysis of nine studies, including seven randomized controlled trials, assessed efficacy of PBM in terms of upper limb volume and pain in the management of lymphedema in patients treated for breast cancer. PBM was associated with moderate level evidence of efficacy in terms of arm volume and pain [38]. It should be noted that the translation of this moderate reduction in arm volume and pain into a clinical benefit for patients was not investigated by the authors. In a randomized placebo-controlled trial including 40 women treated for breast cancer with upper limb lymphedema, the laser PBM protocol $(980 \mathrm{~nm}, 640$ $\mathrm{mW}, 4.89 \mathrm{~J} / \mathrm{cm}^{2}$, spot area $4.9 \mathrm{~cm}^{2}, 10 \mathrm{~min} /$ session, 8 sessions at the axillary level) did not show significant efficacy on quality of life, pain score, grip strength, and arm volume [39]. Recently, a randomized placebo-controlled pilot trial evaluated efficacy at 1 year of a protocol of laser PBM $\left(904 \mathrm{~nm}, 1.5 \mathrm{~J} / \mathrm{cm}^{2}, 1 \mathrm{~min}\right.$ on each of 10 sites in the axilla and part of the homolateral chest wall, 8 to 16 sessions) in combination with manual decongestive therapies in the management of upper limb lymphedema in patients treated for breast cancer. At 1 year, the combination of PBM with decongestive therapies suggested a significant improvement in lymphedema-related symptoms, arm mobility, and emotional distress in these patients [40]. The most recent metaanalysis included seven randomized trials. The main limit was the lack of data regarding PBM parameters. However, the authors concluded that there was strong evidence (three high-quality trials) for the superiority of PBM against placebo in terms of arm circumference and volume reduction and moderate evidence (one high-quality trial) suggesting that PBM would be more effective than placebo for shortterm pain relief [41].

In summary, the systematic review of the literature suggests that PBM is an attractive treatment approach for breast cancer treatment-related upper limb lymphedema. However, due to the moderate level of evidence available, methodologically robust randomized trials are needed, particularly to determine the parameters of PBM.

\section{Safety}

There are no significant adverse events associated with PBM in the literature. However, there is controversy about the potential long-term risks of malignant transformation of healthy cells, or progression and recurrence of the primitive tumor, whose proliferation of tumor cells could be boosted when PBM is applied close to the tumor site [42]. Regarding this issue, in vitro data on the behavior of tumor cells exposed to $\mathrm{PBM}$ are conflicting, and clinical data on the long-term safety of PBM are scarce. A phase III trial with the primary objective of reducing the incidence of acute oral mucositis grade $\geq 3$ randomized 94 patients to either laser PBM $\left(660 \mathrm{~nm}, 100 \mathrm{~mW}, 4 \mathrm{~J} / \mathrm{cm}^{2}\right.$, spot area $0.24 \mathrm{~cm}^{2}, 10 \mathrm{~s} /$ spot, intra-oral) or sham laser in the setting of chemo-radiotherapy for head and neck squamous cell carcinomas. After a relatively short median follow-up of 18 [10-43, 44-48] months, patients treated with PBM appeared to have better locoregional disease control, progression-free survival, and overall survival [15].

To conclude, until robust data are published on its longterm safety, especially on the theoretical risks of transformation into malignant cells or accelerated tumor growth, the clinical use of PBM near areas with known or potential tumor cells should be considered with caution. The patient should be informed of the theoretical benefits and risks of PBM in order to obtain informed consent before treatment.

\section{Discussion}

PBM in the red or infrared spectrum has been shown in randomized controlled trials to be effective in the management of some radiotherapy-related complications, in particular acute mucositis, epithelitis, and upper limb lymphedema. These studies probably even underestimated the magnitude of the benefit of PBM due to incomplete assessment of the costs of these complications. Very recently, a systematic review of the literature concluded that there was evidence in favor of PBM in terms of cost-effectiveness for the prevention and management of cancer treatment-related toxicities, including acute oral mucositis and upper limb lymphedema [43]. Therefore, PBM could be considered as a full-fledged supportive care for patients treated in oncology. Indeed, well beyond the complications of radiotherapy, there is also consistent evidence supporting use of PBM to prevent and manage complications of chemotherapy. While strong evidence including placebo-controlled randomized trials has already 
been published for chemo-induced peripheral neuropathy, convincing data exists for hand-foot syndrome or chemoinduced alopecia. In all cases, a major challenge is now to standardize practices through the publication of guidelines. Detailed protocols of PBM treatment are mandatory in order to optimize its efficacy and guarantee the reproducibility of its outcomes.

PBM is becoming a recognized treatment in many clinical situations when anti-inflammatory effect, analgesic effect, and/or tissue regeneration are expected.

WALT, in close collaboration with MASCC and ISOO, is defining optimal technical and dosimetric parameters in all indications, with collaborative guidelines and recommendations (especially for PBM using modern LED devices).

Regarding the different types of PBM applicators for cancer patients, we have the option of commercially available extra-oral devices and intra-oral devices, targeting structures such as cutaneous and oral mucosal surfaces, respectively. Also, we must remember the fact that, while using an extraoral device for the application of PBM, to a certain extent (with wavelengths around $830 \mathrm{~nm}$, not with 630-660 nm), we may be able to indirectly reach intra-oral surfaces such as (for head and neck region) the oral mucosa, vestibule, and inner epithelial surfaces of the lips in a dentate subject. This proves that a combination of the above two devices must be considered while managing the head and neck radiotherapy-induced side effects but not necessary in chemotherapy induced intra-oral effects.

Finally, we believe that the following parameters should become mandatory while considering PBM in all its indications. The parameters to be considered include wavelength $(\mathrm{nm})$, power $(\mathrm{mW}), \mathrm{J} / \mathrm{cm}^{2}$ per point (or "dose"), energy density, spot size, power density $\left(\mathrm{mW} / \mathrm{cm}^{2}\right)$, and laser machine calibration. Treatment characteristics should include the total number of $\mathrm{J} / \mathrm{cm}^{2}$ in any single laser session, the total number of sessions, the frequency of sessions (treatment protraction), the site(s) of treatment, and some precision regarding laser administration (contact pressure treatment, application over single area at one time than scanning motion, preparation of the mucosal or cutaneous surface) and most importantly a well-trained individual such as cancer specialist who could assess the region of interest, lesions to treat, and to grade them accordingly.

Regarding equipment required for an optimal PBM treatment, we have to choose companies that can certify PBM dose delivery and control this dose delivery in routine practice.

External LED devices are cheaper than laser diodes, with the same efficiency when high-quality LEDS are used (with unidirectional beam; monochromatic or polychromatic device, with one or a range of available wavelengths). More generally, external devices are more versatile than intra-oral (or intra-cavitary) devices, and they are now totally validated in terms of calibration and clinical efficacy.

In all cases, maintenance of the machines, with regular controls, is needed.

WALT and other laser societies have to play a key role in the quality control of commercial devices.

Regarding PBM users, physicians have to prescribe PBM treatments, but they can delegate the realization of daily PBM delivery to the patient to a nurse or a radiotherapist.

Prescription is a medical task: knowing the target and the pathology that have to be treated conduct to the right choice in PBM device and in PBM parameters (wavelength, dose, etc.).

The first university diploma, which validate a 1-year training in "PBM in Cancer Supportive Care", was created in France in 2020 (University of Paris-Saclay, Gustave Roussy Institute) with a great success.

Regarding cost-effectiveness of PBM compared with other supportive care treatments, we have few comparative data that include the use of modern PBM material and new quality control criteria (with a much better expected income and reliability compared with old devices). These comparative studies are now initiated in several countries and should confirm in the near future this cost-effectiveness: PBM material is not expensive, the main limiting factor being the cost of salaries (people using devices, 10 to $20 \mathrm{mn}$ per patient). Moreover, reduction in the number and duration of hospitalizations, reduction of enteral or parenteral nutrition, and reduction of the use of morphinics, for example, were largely documented in several studies testing PBM for oral mucositis in cancer patients, with a significant decrease of treatment cost per patient.

Finally, with all this technical and clinical progress, PBM is entering a new era of maturity and evidence-based clinical use that should allow PBM to be fully considered as a part of "mainstream medicine"!

\section{Declarations}

Conflict of interest The authors declare no competing interests.

\section{References}

1. Anders JJ, Arany PR, Baxter GD, Lanzafame RJ (2019) Lightemitting diode therapy and low-level light therapy are photobiomodulation therapy. Photobiomodul Photomed Laser Surg 37:63-65. https://doi.org/10.1089/photob.2018.4600

2. Zecha JAEM, Raber-Durlacher JE, Nair RG, Epstein JB, Sonis ST, Elad S et al (2016) Low level laser therapy/photobiomodulation in the management of side effects of chemoradiation therapy in head and neck cancer: part 1: mechanisms of action, dosimetric, 
and safety considerations. Support Care Cancer 24:2781-2792. https://doi.org/10.1007/s00520-016-3152-z

3. Cotler HB (2015) A NASA discovery has current applications in orthopaedics. Curr Orthop Pract 26:72-74. https://doi.org/10. 1097/BCO.0000000000000196

4. Heiskanen V, Hamblin MR (2018) Photobiomodulation: lasers vs. light emitting diodes? Photochem Photobiol Sci 17:1003-17. https://doi.org/10.1039/c8pp90049c

5. Chung H, Dai T, Sharma SK, Huang Y-Y, Carroll JD, Hamblin MR (2012) The nuts and bolts of low-level laser (light) therapy. Ann Biomed Eng 40:516-533. https://doi.org/10.1007/ s10439-011-0454-7

6. Frigo L, Fávero GM, Lima HJC, Maria DA, Bjordal JM, Joensen $\mathrm{J}$ et al (2010) Low-level laser irradiation (InGaAlP-660 nm) increases fibroblast cell proliferation and reduces cell death in a dose-dependent manner. Photomed Laser Surg 28(Suppl 1):S151156. https://doi.org/10.1089/pho.2008.2475

7. Zecha JAEM, Raber-Durlacher JE, Nair RG, Epstein JB, Elad S, Hamblin MR et al (2016) Low-level laser therapy/photobiomodulation in the management of side effects of chemoradiation therapy in head and neck cancer: part 2: proposed applications and treatment protocols. Support Care Cancer 24:2793-2805. https:// doi.org/10.1007/s00520-016-3153-y

8. Zadik Y, Arany PR, Fregnani ER, Bossi P, Antunes HS, Bensadoun R-J et al (2019) Systematic review of photobiomodulation for the management of oral mucositis in cancer patients and clinical practice guidelines. Support Care Cancer 27:3969-3983. https://doi.org/10.1007/s00520-019-04890-2

9. Lalla RV, Treister N, Sollecito T, Schmidt B, Patton LL, Mohammadi $\mathrm{K}$ et al (2017) Oral complications at six months after radiation therapy for head and neck cancer. Oral Dis 23:1134-1143. https://doi.org/10.1111/odi.12710

10. Gouvêa de Lima A, Villar RC, de Castro G, Antequera R, Gil E, Rosalmeida MC et al (2012) Oral mucositis prevention by lowlevel laser therapy in head-and-neck cancer patients undergoing concurrent chemoradiotherapy: a phase III randomized study. Int J Radiat Oncol Biol Phys 82:270-5. https://doi.org/10.1016/j.ijrobp. 2010.10.012

11. Gautam AP, Fernandes DJ, Vidyasagar MS, Maiya AG, Vadhiraja BM (2012) Low level laser therapy for concurrent chemoradiotherapy induced oral mucositis in head and neck cancer patients - a triple blinded randomized controlled trial. Radiother Oncol 104:349-354. https://doi.org/10.1016/j.radonc.2012.06.011

12. Gautam AP, Fernandes DJ, Vidyasagar MS, Maiya GA (2012) Low level helium neon laser therapy for chemoradiotherapy induced oral mucositis in oral cancer patients - a randomized controlled trial. Oral Oncol 48:893-897. https://doi.org/10.1016/j. oraloncology.2012.03.008

13. Gautam AP, Fernandes DJ, Vidyasagar MS, Maiya AG, Nigudgi S (2013) Effect of low-level laser therapy on patient reported measures of oral mucositis and quality of life in head and neck cancer patients receiving chemoradiotherapy-a randomized controlled trial. Support Care Cancer 21:1421-1428. https://doi.org/10.1007/ s00520-012-1684-4

14. Oton-Leite AF, Elias LSA, Morais MO, Pinezi JCD, Leles CR, Silva MAGS et al (2013) Effect of low level laser therapy in the reduction of oral complications in patients with cancer of the head and neck submitted to radiotherapy. Spec Care Dentist 33:294300. https://doi.org/10.1111/j.1754-4505.2012.00303.x

15. Antunes HS, Herchenhorn D, Small IA, Araújo CMM, Viégas CMP, Cabral E et al (2013) Phase III trial of low-level laser therapy to prevent oral mucositis in head and neck cancer patients treated with concurrent chemoradiation. Radiother Oncol 109:297-302. https://doi.org/10.1016/j.radonc.2013.08.010

16. Gautam AP, Fernandes DJ, Vidyasagar MS, Maiya AG, Guddattu V (2015) Low level laser therapy against radiation induced oral mucositis in elderly head and neck cancer patients-a randomized placebo controlled trial. J Photochem Photobiol B 144:51-56. https://doi.org/10.1016/j.jphotobiol.2015.01.011

17. Oton-Leite AF, Silva GBL, Morais MO, Silva TA, Leles CR, Valadares MC et al (2015) Effect of low-level laser therapy on chemoradiotherapy-induced oral mucositis and salivary inflammatory mediators in head and neck cancer patients. Lasers Surg Med 47:296-305. https://doi.org/10.1002/lsm.22349

18. Campos TM, do Prado Tavares Silva CA, Sobral APT, Sobral SS, Rodrigues MFSD, Bussadori SK et al (2020) Photobiomodulation in oral mucositis in patients with head and neck cancer: a systematic review and meta-analysis followed by a cost-effectiveness analysis. Support Care Cancer 28:5649-59. https://doi.org/10. 1007/s00520-020-05613-8

19. de Pauli PM, Alves CGB, Fontes EK, Lopes MA, Ribeiro ACP, Brandão TB et al (2019) Is photobiomodulation therapy effective in reducing pain caused by toxicities related to head and neck cancer treatment? A systematic review Support Care Cancer 27:4043-4054. https://doi.org/10.1007/s00520-019-04939-2

20. Lalla RV, Bowen J, Barasch A, Elting L, Epstein J, Keefe DM et al (2014) MASCC/ISOO clinical practice guidelines for the management of mucositis secondary to cancer therapy. Cancer 120:1453-1461. https://doi.org/10.1002/cncr.28592

21. Elad S (2020) The MASCC/ISOO mucositis guidelines 2019: the second set of articles and future directions. Support Care Cancer 28:2445-2447. https://doi.org/10.1007/s00520-019-05153-w

22. Wong RKS, Bensadoun R-J, Boers-Doets CB, Bryce J, Chan A, Epstein JB et al (2013) Clinical practice guidelines for the prevention and treatment of acute and late radiation reactions from the MASCC Skin Toxicity Study Group. Support Care Cancer 21:2933-2948. https://doi.org/10.1007/s00520-013-1896-2

23. Fife D, Rayhan DJ, Behnam S, Ortiz A, Elkeeb L, Aquino L et al (2010) A randomized, controlled, double-blind study of light emitting diode photomodulation for the prevention of radiation dermatitis in patients with breast cancer. Dermatol Surg 36:19211927. https://doi.org/10.1111/j.1524-4725.2010.01801.x

24. Censabella S, Claes S, Robijns J, Bulens P, Mebis J (2016) Photobiomodulation for the management of radiation dermatitis: the DERMIS trial, a pilot study of MLS( $\left({ }^{\circledR}\right)$ laser therapy in breast cancer patients. Support Care Cancer 24:3925-3933. https://doi. org/10.1007/s00520-016-3232-0

25. Robijns J, Censabella S, Claes S, Pannekoeke L, Bussé L, Colson D et al (2018) Prevention of acute radiodermatitis by photobiomodulation: a randomized, placebo-controlled trial in breast cancer patients (TRANSDERMIS trial). Lasers Surg Med. https://doi. org/10.1002/lsm.22804

26. Robijns J, Lodewijckx J, Claes S, Van Bever L, Pannekoeke L, Censabella S et al (2021) Photobiomodulation therapy for the prevention of acute radiation dermatitis in head and neck cancer patients (DERMISHEAD trial). Radiother Oncol 158:268-275. https://doi.org/10.1016/j.radonc.2021.03.002

27. Robijns J, Lodewijckx J, Bensadoun R-J, Mebis J (2020) A narrative review on the use of photobiomodulation therapy for the prevention and management of acute radiodermatitis: proposed mechanisms, current clinical outcomes, and preliminary guidance for clinical studies. Photobiomodul Photomed Laser Surg 38:332-339. https://doi.org/10.1089/photob.2019.4761

28. Russi EG, Corvò R, Merlotti A, Alterio D, Franco P, Pergolizzi $S$ et al (2012) Swallowing dysfunction in head and neck cancer patients treated by radiotherapy: review and recommendations of the supportive task group of the Italian Association of Radiation Oncology. Cancer Treat Rev 38:1033-1049. https://doi.org/10. 1016/j.ctrv.2012.04.002

29. Nutting CM, Morden JP, Harrington KJ, Urbano TG, Bhide SA, Clark C et al (2011) Parotid-sparing intensity modulated versus conventional radiotherapy in head and neck cancer (PARSPORT): 
a phase 3 multicentre randomised controlled trial. Lancet Oncol 12:127-136

30. Epstein JB, Robertson M, Emerton S, Phillips N, StevensonMoore P (2001) Quality of life and oral function in patients treated with radiation therapy for head and neck cancer. Head Neck 23:389-398. https://doi.org/10.1002/hed.1049

31. Cowen D, Tardieu C, Schubert M, Peterson D, Resbeut M, Faucher C et al (1997) Low energy helium-neon laser in the prevention of oral mucositis in patients undergoing bone marrow transplant: results of a double blind randomized trial. Int J Radiat Oncol Biol Phys 38:697-703. https://doi.org/10.1016/s03603016(97)00076-x

32. Louzeiro GC, Cherubini K, de Figueiredo MAZ, Salum FG (2020) Effect of photobiomodulation on salivary flow and composition, xerostomia and quality of life of patients during head and neck radiotherapy in short term follow-up: a randomized controlled clinical trial. J Photochem Photobiol B 209:111933. https://doi. org/10.1016/j.jphotobiol.2020.111933

33. Saleh J, Figueiredo MAZ, Cherubini K, Braga-Filho A, Salum FG (2014) Effect of low-level laser therapy on radiotherapy-induced hyposalivation and xerostomia: a pilot study. Photomed Laser Surg 32:546-552. https://doi.org/10.1089/pho.2014.3741

34. Heiskanen V, Zadik Y, Elad S (2020) Photobiomodulation therapy for cancer treatment-related salivary gland dysfunction: a systematic review. Photobiomodul Photomed Laser Surg 38:340-347. https://doi.org/10.1089/photob.2019.4767

35. Louzeiro GC, da Silveira Teixeira D, Cherubini K, de Figueiredo MAZ, Salum FG (2020) Does laser photobiomodulation prevent hyposalivation in patients undergoing head and neck radiotherapy? A systematic review and meta-analysis of controlled trials. Crit Rev Oncol Hematol 156:103115. https://doi.org/10.1016/j. critrevonc.2020.103115

36. DiSipio T, Rye S, Newman B, Hayes S (2013) Incidence of unilateral arm lymphoedema after breast cancer: a systematic review and meta-analysis. Lancet Oncol 14:500-515. https://doi.org/10. 1016/S1470-2045(13)70076-7

37. Hwang JM, Hwang JH, Kim TW, Lee SY, Chang HJ, Chu IH (2013) Long-term effects of complex decongestive therapy in breast cancer patients with arm lymphedema after axillary dissection. Ann Rehabil Med 37:690-697. https://doi.org/10.5535/ arm.2013.37.5.690

38. Smoot B, Chiavola-Larson L, Lee J, Manibusan H, Allen DD (2015) Effect of low-level laser therapy on pain and swelling in women with breast cancer-related lymphedema: a systematic review and meta-analysis. J Cancer Surviv 9:287-304. https:// doi.org/10.1007/s11764-014-0411-1

39. Storz MA, Gronwald B, Gottschling S, Schöpe J, Mavrova R, Baum S (2017) Photobiomodulation therapy in breast cancerrelated lymphedema: a randomized placebo-controlled trial. Photodermatol Photoimmunol Photomed 33:32-40. https://doi. org/10.1111/phpp.12284

40. Kilmartin L, Denham T, Fu MR, Yu G, Kuo T-T, Axelrod D et al (2020) Complementary low-level laser therapy for breast cancerrelated lymphedema: a pilot, double-blind, randomized, placebocontrolled study. Lasers Med Sci 35:95-105. https://doi.org/10. 1007/s10103-019-02798-1

41. Baxter GD, Liu L, Petrich S, Gisselman AS, Chapple C, Anders JJ et al (2017) Low level laser therapy (photobiomodulation therapy) for breast cancer-related lymphedema: a systematic review. BMC Cancer 17:833. https://doi.org/10.1186/s12885-017-3852-x

42. Sonis ST, Hashemi S, Epstein JB, Nair RG, Raber-Durlacher JE (2016) Could the biological robustness of low level laser therapy (photobiomodulation) impact its use in the management of mucositis in head and neck cancer patients. Oral Oncol 54:7-14. https://doi.org/10.1016/j.oraloncology.2016.01.005

43. Kauark-Fontes E, Rodrigues-Oliveira L, Epstein JB, Faria KM, Araújo ALD, Gueiros LAM et al (2021) Cost-effectiveness of photobiomodulation therapy for the prevention and management of cancer treatment toxicities: a systematic review. Support Care Cancer 29:2875-2884. https://doi.org/10.1007/ s00520-020-05949-1

Publisher's Note Springer Nature remains neutral with regard to jurisdictional claims in published maps and institutional affiliations. 